\title{
Cerebral vasculitis and lateral rectus palsy - two rare central nervous system complications of dengue fever: two case reports and review of the literature
}

\author{
H. M. M. Herath ${ }^{1 *}$, J. S. Hewavithana ${ }^{2}$, C. M. De Silva ${ }^{2}$, O. A. R. Kularathna ${ }^{2}$ and N. P. Weerasinghe ${ }^{3}$
}

\begin{abstract}
Background: Dengue fever is a common mosquito-borne viral illness with a clinical spectrum ranging from a simple febrile illness to potentially life-threatening complications such as dengue hemorrhagic fever and dengue shock syndrome. Dengue infection can affect many organs, including the central nervous system. The neurological manifestations reported in dengue infections are meningitis, encephalitis, stroke, acute disseminated encephalomyelitis, and Guillain-Barré syndrome.

Case presentation: We report the cases of two interesting patients with confirmed dengue infection who presented with complications of possible central nervous system vasculitis and cranial nerve palsy. The first patient was a 53-year-old previously healthy Singhalese woman who developed acute-onset slurring of speech and ataxia with altered sensorium 1 day after recovery from a critical period of dengue hemorrhagic fever. Subsequent investigations revealed evidence of encephalopathy with brainstem ischemic infarctions. Her clinical picture was compatible with central nervous system vasculitis. She was treated successfully with intravenous steroids and had a full functional recovery. The second patient was a middle-aged Singhalese woman who had otherwise uncomplicated dengue infection. She developed binocular diplopia on day 4 of fever. An ocular examination revealed a convergent squint in the left eye with lateral rectus palsy but no other neurological manifestation.
\end{abstract}

Conclusions: Central nervous system vasculitis due to dengue infection is a very rare phenomenon, and to the best of our knowledge, only one case of central nervous system vasculitis has been reported to date, in a patient of pediatric age. Cranial nerve palsy related to dengue infection is also rare, and only a few cases of isolated abducens nerve palsy have been reported to date. The two cases described in this report illustrate the rare but important central nervous system manifestations of dengue fever and support the fact that the central nervous system is one of the important systems that can be affected in patients with dengue infection.

Keywords: Dengue infection, Cerebral vasculitis, Encephalitis, Cranial nerve palsy, Neurological complications

\section{Background}

Dengue fever is a common arboviral infection in tropical and subtropical areas of the world. It is transmitted by the hematophagous mosquito Aedes aegypti [1]. Around 2.5 billion people in more than 100 countries are at risk of dengue infection, with about 100 million cases of

\footnotetext{
* Correspondence: herathtp@gmail.com

'Department of Medicine, Faculty of Medicine, University of Ruhuna, P.O. Box 70, Galle, Sri Lanka

Full list of author information is available at the end of the article
}

symptomatic dengue occurring annually [2]. Being a tropical country with high rainfall, Sri Lanka has experienced frequent dengue outbreaks for the last several decades. In 2017 , over 100,000 cases of dengue infection were reported in Sri Lanka [3]. Dengue infection can be caused by one of four distinct, antigenically related serotypes: DEN-1, DEN-2, DEN-3, and DEN-4 [1].

The clinical presentation of dengue infection ranges from mild clinical febrile illness to potentially lifethreatening dengue hemorrhagic fever (DHF) and dengue 
shock syndrome (DSS) [4]. The main cause of death in dengue infection is either DHF or DSS, both of which are due to increased capillary permeability resulting in plasma leakage [5]. Apart from these complications, dengue infection is known to cause multiple organ involvement, including the central nervous system (CNS) [6-10]. Neurological complications in dengue infection can be explained by three different pathogenic mechanisms: (1) direct invasion of the CNS, leading to encephalitis, meningitis and myelitis; (2) systemic complications resulting in encephalopathy and stroke; and (3) para- or postinfectious immune-mediated effects, such as acute disseminated encephalomyelitis (ADEM), Guillain-Barré syndrome, and optic neuritis $[7,11]$.

We report two cases of patients with dengue infection associated with CNS complications. The first patient had possible CNS vasculitis related to dengue, and the second had isolated cranial nerve (abducens nerve) palsy. These two cases are of importance because, to the best of our knowledge, CNS vasculitis triggered by dengue infection has not been described previously in the literature. Furthermore, only three cases of isolated cranial nerve palsy related to dengue infection have been reported previously.

\section{Case presentation}

\section{Patient 1}

An otherwise healthy 53-year-old Singhalese woman who was an office worker from Southern Province, Sri Lanka, presented to a local hospital with an acute febrile illness with no apparent focus of infection. She had no significant past medical illnesses and did not smoke or consume alcohol. Three days prior to her hospital admission, she developed high fever with chills, myalgia, and moderate headache. She was conscious and rational with no hemodynamic compromise. Her full blood count revealed a hemoglobin level of $13.6 \mathrm{~g} / \mathrm{dl}$, packed cell volume (PCV) of $37.9 \%$, white blood cell count of $4.2 \times 10^{3} / \mathrm{mm}^{3}$, and platelet count of $105 \times 10^{3} / \mathrm{mm}^{3}$. The result of her nonstructural protein 1 (NS1) antigen test (enzyme-linked immunosorbent assay) was positive. Her liver transaminases were elevated with aspartate aminotransferase (AST) of $161 \mathrm{U} /$ $\mathrm{L}$ and alanine aminotransferase (ALT) of $113 \mathrm{U} / \mathrm{L}$. In the next 48 hours, she was observed in the local hospital with serial full blood count measurements, which showed a gradual reduction of her platelet count to $41 \times 10^{3} / \mathrm{mm}^{3}$ on the fifth day of her illness, at which point her PCV had risen to $42 \%$. She had evidence of free fluid in the hepatorenal pouch on an ultrasound scan, indicating progression into the critical phase of DHF. She was observed and managed in the next 48 hours of the critical phase according to the established national guidelines on dengue management [12]. She went through the critical phase without any significant complication, such as bleeding, shock, or multiorgan failure. Following resolution of the critical phase, she was afebrile and clinically improving. Her PCV dropped from $42 \%$ to $36.5 \%$, and her platelets showed a gradual increase to $141 \times$ $10^{3} / \mathrm{mm}^{3}$. However, on postcritical day 1 , she experienced acute-onset slurring of speech, deviation of the mouth, difficulty in swallowing, and unsteadiness in gait. At this juncture, she had no fever, headache, or seizures. An urgent noncontrast computed tomographic scan did not demonstrate any particular abnormality in the brain and brainstem. The patient was transferred to our unit for further evaluation and management.

On admission to our unit, the patient was hemodynamically stable with a blood pressure of $112 / 74 \mathrm{mmHg}$ and pulse rate of 84 beats/minute. She was afebrile but was slightly drowsy with a Glasgow Coma Scale score of 13/15. She had right-sided lower motor-type facial nerve (cranial nerve VII) palsy with ipsilateral palatal weakness, horizontal nystagmus with fast component to the right, and mild weakness in the left upper and lower limbs with normal reflexes and sensory and plantar responses. The rest of the examination, including funduscopy, was unremarkable. Subsequent laboratory investigations, including septic screening with blood culture and mycoplasma serology, serum electrolytes, and liver and renal function, did not reveal any significant abnormalities. Apart from leukopenia (total white cell count of $3400 / \mathrm{mm}^{3}, 42 \%$ neutrophils, 56\% lymphocytes), a repeat full blood count showed no significant abnormalities. The diagnosis of dengue infection was reconfirmed by the presence of dengue-specific immunoglobulin G (IgG) and IgM antibodies in serum on day 7 of the patient's illness (ELISA method). Analysis of the patient's cerebrospinal fluid (CSF) revealed a normal protein level of $53 \mathrm{~g} / \mathrm{L}$, a normal glucose level, and no pleocytosis. An electroencephalogram (EEG) showed features of encephalitis (Fig. 1). Magnetic resonance imaging (MRI) of the brain revealed multiple infarctions involving the pons and medulla regions (Fig. 2).

The patient was treated with intravenous dexamethasone to reduce cerebral inflammation. Supportive measures, including physiotherapy and speech therapy, were initiated. She had gradual but progressive improvement over the next few weeks and had a full functional recovery around 1 month after the illness. CNS vasculitis due to dengue infection was the presumptive diagnosis, based on the clinical presentation of acute onset of focal neurological signs with evidence of brainstem infarction and encephalopathy.

\section{Patient 2}

A 57-year-old previously healthy Singhalese woman who was a schoolteacher was admitted to our unit with a 


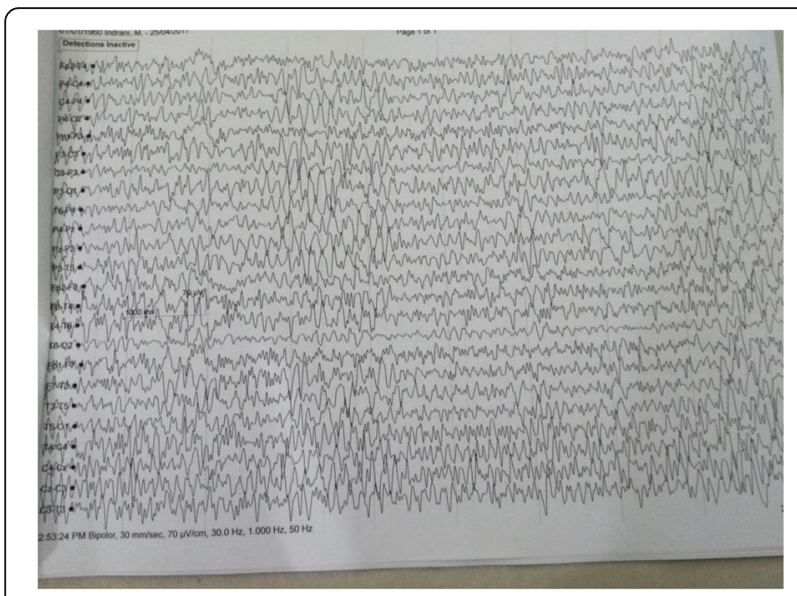

Fig. 1 Electroencephalogram showing generalized spike and wave discharges and periodicity compatible with encephalopathy

history of fever, headache, malaise, nausea, and anorexia of 3 days' duration. She did not smoke or use alcohol or recreational drugs. On examination, she had a temperature of $39.5{ }^{\circ} \mathrm{C}$ and was hemodynamically stable with a pulse rate of 66 beats/minute and blood pressure of $112 / 66 \mathrm{mmHg}$ with no significant postural drop. The rest of her examination was unremarkable. Her full blood count revealed a total leukocyte count of $4100 / \mathrm{mm}^{3}$ with $42 \%$ lymphocytes, hemoglobin of $13.4 \mathrm{~g} / \mathrm{dl}, \mathrm{PCV}$ of $40 \%$, platelet count of $94,000 / \mathrm{mm}^{3}$, and elevated liver transaminases (ALT of $77 \mathrm{U} / \mathrm{L}$ and AST of $86 \mathrm{U} / \mathrm{L})$. Dengue infection was confirmed by detection of the NS1 antigen.

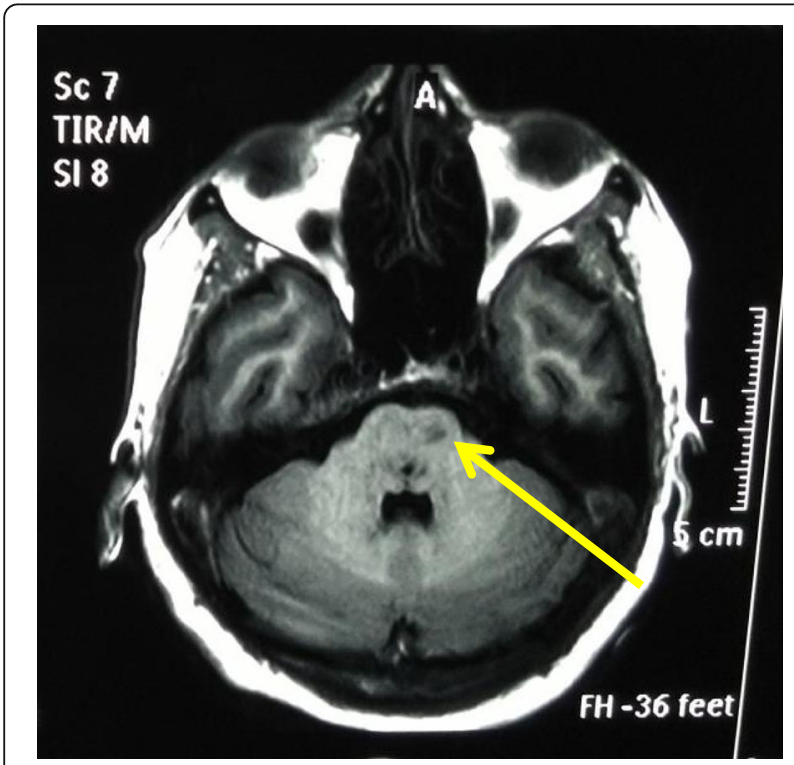

Fig. 2 Magnetic resonance imaging scan of the brainstem showing multiple infarctions involving the pons and medulla regions (arrow)
The patient was started on treatment for uncomplicated dengue fever. However, on the second day of her hospital admission, she started complaining of bilateral diplopia, which worsened upon left gaze. She did not complain of headache, and her consciousness level was not reduced at this stage. An ocular examination revealed a convergent squint in the left eye with evidence of isolated lateral rectus weakness. The results of the rest of the CNS examination, including the vision and fundus of both eyes, were normal. The patient was investigated with an MRI scan of the brain, which ruled out a spaceoccupying lesion or any other significant pathology. During the patient's hospital stay, her platelet count dropped gradually to $44,000 / \mathrm{mm}^{3}$ on the fifth day of fever, before it started to rise. Apart from isolated lateral rectus weakness, she did not develop any other complications, such as plasma leakage, shock, hemorrhage, or liver failure.

She was managed with intravenous and oral fluids according to the national guideline for the management of dengue fever [12]. She recovered from dengue fever and was discharged from the hospital on day 7 of her illness. She had evidence of improvement of her lateral rectus palsy at the time of discharge, and it completely resolved within 4 weeks without any specific treatment.

\section{Discussion}

The two patients with dengue infection whom we describe in this report, one with possible CNS vasculitis and the other with isolated cranial nerve (abducens nerve) palsy, had extremely rare CNS manifestations of dengue infection. Particularly, CNS vasculitis triggered by dengue infection has not been described in the literature previously, to the best of our knowledge. Furthermore, only three cases of isolated cranial nerve palsy related to dengue infection have been reported previously.

Dengue infections pose a significant health challenge in affected countries in the tropics [2]. CNS manifestations are increasingly recognized and can cause diagnostic and management dilemmas [13]. A wide variety of CNS manifestations of dengue have been described, ranging from meningitis [14-16] to immune-mediated disease such as Guillain-Barré syndrome [17, 18] and optic neuritis [19]. CNS involvement of dengue infection was first described by Sanguansermsri et al. in 1976 in a patient who presented with encephalopathy [20]. Since then, many different types of CNS involvement have been described. Even though the pathogenesis of CNS involvement of dengue infection is still poorly understood, direct viral invasion, autoimmune reaction, and metabolic and hemorrhagic disturbances are widely considered as possible mechanisms. Murthy categorized CNS manifestations in dengue infection into three groups [21]: (1) CNS involvement due to direct neurotropic effects such as 
meningitis, encephalitis, and myelitis; (2) CNS involvement due to metabolic complications such as encephalopathy, hypokalemic paralysis, and stroke; and (3) postviral immune-mediated CNS complications such as encephalomyelitis, optic neuritis, and Guillain-Barré syndrome [21].

Based on patient 1's presentation and investigations, CNS vasculitis was considered as the most likely diagnosis. Most patients with isolated CNS vasculitis typically have headache and features of encephalopathy, with evidence of multiple cerebral infarctions [22]. Patient 1 developed ischemic stroke, as evidenced by acute onset of focal neurological symptoms (dysarthria and ataxia) and signs of brainstem lesions (such as palatal palsy and lower motor facial nerve palsy) with evidence of brainstem infarctions involving the pons and medulla regions on an MRI scan. In addition, patient 1 had clear evidence of encephalopathy, as indicated by altered sensorium and typical EEG abnormalities observed in encephalopathy. Alternative causes for patient 1's encephalopathy were considered unlikely, given that there was no evidence of hemodynamic compromise, metabolic derangement such as hypoglycemia, electrolyte imbalance, or liver failure. This was further confirmed by the presence of a normal CSF study. Dengue encephalitis was considered as one of the possibilities. However, the combination of stroke with multiple infarctions, encephalopathy with altered sensorium, headache, and absence of CNS pleocytosis made CNS vasculitis more likely. Furthermore, the main features of dengue encephalitis, such as seizures and CNS pleocytosis, were absent in patient 1 [23]. Even though CNS pleocytosis may be absent in some patients with dengue encephalitis, it is a well-recognized feature of the disease $[13,15,23]$. For example, the diagnostic criteria for dengue encephalitis proposed by Carod-Artal et al. included CSF pleocytosis [11].

There are many challenges in making a definitive diagnosis of isolated cerebral vasculitis [22]. The lack of uniform consensus regarding diagnostic criteria, as well as diagnostic limitations of specific investigations such as angiography and imaging, present important challenges. Angiography was not considered in patient 1 because she responded to the treatment well and she was unwilling to undergo further testing due to her prolonged hospital stay. CNS vasculitis due to dengue infection is a very rare phenomenon, and to the best of our knowledge, only one case of CNS vasculitis has been reported to date, in a patient of pediatric age [24]. As far as we are aware, there are no previous cases of CNS vasculitis reported in adults with dengue.

Regarding treatment, the mainstay of treatment for dengue infection and its associated CNS complications is supportive therapy based on locally available guidelines [7, 12, 23, 25]; however, steroids may have a place in treating some of the immune-mediated CNS complications. There is some evidence that CNS complications such as ADEM [26, 27] and myelitis [25, 28] have a favorable response to steroid therapy. Patient 1 also had a good response to an intravenous steroid (dexamethasone), which may suggest that an underlying immunemediated pathological process was the most likely reason for the CNS manifestation.

Patient 2 had isolated sixth cranial nerve palsy that developed on the fourth day of her illness. Cranial nerve palsy associated with dengue infection is also relatively rare, with only a few cases reported previously [8, 29]. Optic neuropathy [19], oculomotor nerve palsy [30], and facial nerve palsy $[9,31]$ associated with dengue infection have been described previously. Abducens nerve palsy has also been described, but only in a few cases $[8,9,29]$. All reported cases of abducens nerve palsy were in male patients, and none of them had features of DHF (plasma leakage) or DSS. Most patients developed abducens nerve palsy roughly 1 week after the onset of fever. However, patient 2 and the patient reported by Mazliha et al. [9] developed abducens nerve palsy within the first 5 days of the onset of fever (Table 1). Even though some authors have suggested that the underlying pathophysiology behind cranial palsy could be due to an immune-mediated mechanism, direct viral invasion of cranial nerves is more likely, considering the fact that some patients developed abducens nerve palsy at an early stage of their illness (days 2-4). The fact that all patients recovered fairly rapidly without any specific therapies such as steroids favors direct viral invasion over immunemediated mechanisms [7, 9].

Specific therapies, such as steroids or immunoglobulins, are not generally required in the management of dengue-related abducens nerve palsy $[8,9,29]$. Patient 2 was managed with supportive therapy with fluid and paracetamol during the acute stage. She had a complete

Table 1 Characteristics of previously reported patients with abducens nerve palsy

\begin{tabular}{|c|c|c|c|c|c|c|}
\hline Study & Patient age (years) & Sex & $\begin{array}{l}\text { Day of onset of } \\
\text { mononeuropathy in } \\
\text { relation to fever }\end{array}$ & Type of mononeuropathy & $\begin{array}{l}\text { Associated with } \\
\text { DHF or DSS }\end{array}$ & $\begin{array}{l}\text { Associated with other } \\
\text { CNS manifestation }\end{array}$ \\
\hline Mishra, et al. [29] & 52 & Male & Day 8 & Lateral rectus palsy & No & No \\
\hline Shivanthan MC et al. & 29 & Male & Day 7 & Lateral rectus palsy & No & No \\
\hline Mazliha, et al. [9] & 40 & Male & Day 2 & Lateral rectus & No & No \\
\hline
\end{tabular}

Abbreviations: CNS Central nervous system, DHF Dengue hemorrhagic fever, DSS Dengue shock syndrome 
recovery from the nerve palsy within the first 4 weeks of the illness. Similar to patient 2, other patients with dengue-associated cranial nerve palsies, including abducens nerve palsy, Bell's palsy, and oculomotor nerve palsy, have been reported to improve without any specific therapy $[8,9]$. The prognosis for dengue-related cranial nerve palsies is good, and complete recovery was observed in all previously reported cases $[8,9,25]$.

\section{Conclusions}

Even though most cases of dengue infection have a selflimiting course, some patients develop devastating and life-threatening complications such as DHF and DSS. In addition to these two complications, dengue infection can affect many other organ systems, including the CNS. Even though a wide variety of CNS manifestations of dengue infection have been reported, CNS vasculitis associated with dengue infection has not been described previously among adults. To the best of our knowledge, we report the first case of CNS vasculitis in an adult patient with dengue infection. Furthermore, abducens nerve palsy in the setting of fever in a tropical country such as Sri Lanka could very well be due to dengue infection, and dengue infection should be considered as a cause of abducens nerve palsy.

\section{Abbreviations}

ADEM: Acute disseminated encephalomyelitis; ALT: Alanine aminotransferase; AST: Aspartate aminotransferase; CNS: Central nervous system; CSF: Cerebrospinal fluid; DHF: Dengue hemorrhagic fever; DSS: Dengue shock syndrome; EEG: Electroencephalogram; ELISA: Enzyme-linked immunosorbent assay; Ig: Immunoglobulin; MRI: Magnetic resonance imaging; NS1: Nonstructural protein 1; PCV: Packed cell volume

\section{Acknowledgements}

The authors acknowledge the two patients profiled in this case report for permitting us to publish this case report. The authors also acknowledge Dr. Krishantha Jayasekara, consultant physician at Balapitiya Base Hospital, and all the staff of the university medical unit of Karapitiya Teaching Hospital for their assistance in investigating and managing the two patients presented in this case report.

\section{Funding}

No funding was received for this report.

\section{Availability of data and materials}

Data sharing is not applicable to this article, because no datasets were generated or analyzed. The two reported patients' hospital records are not shared, because they contain personally identifiable information.

\section{Authors' contributions}

$\mathrm{HMMH}$ was involved in patient diagnosis and management and in writing the manuscript. JSH, CMDS, and OARK were involved in investigations and writing the manuscript. NPW was involved in writing the manuscript. All authors read and approved the final manuscript.

\section{Ethics approval and consent to participate}

Not applicable.

\section{Consent for publication}

Written informed consent was obtained from the patients for publication of this case report and any accompanying images. Copies of the written consent are available for review by the Editor-in-Chief of this journal.

\section{Competing interests}

The authors declare that they have no competing interests.

\section{Publisher's Note}

Springer Nature remains neutral with regard to jurisdictional claims in published maps and institutional affiliations.

\section{Author details}

${ }^{1}$ Department of Medicine, Faculty of Medicine, University of Ruhuna, P.O. Box 70, Galle, Sri Lanka. '2University Unit, Karapitiya Teaching Hospital, Galle, Sri Lanka. ${ }^{3}$ Department of Microbiology, Faculty of Medicine, University of Ruhuna, Galle, Sri Lanka.

Received: 11 October 2017 Accepted: 19 February 2018

Published online: 19 April 2018

\section{References}

1. Murray NEA, Quam MB, Wilder-Smith A. Epidemiology of dengue: past, present and future prospects. Clin Epidemiol. 2013;5:299-309.

2. Fredericks AC, Fernandez-Sesma A. The burden of dengue and chikungunya worldwide: implications for the southern United States and California. Ann Glob Health. 2014;80(6):466-75.

3. Epidemiology Unit, Ministry of Health, Sri Lanka. Dengue update. http:// www.epid.gov.Ik/web/index.php?option=com_content\&view=article\&id= 171\&ltemid=487\&lang=en. Accessed 10 Dec 2017.

4. Gubler DJ. Dengue and dengue hemorrhagic fever. Clin Microbiol Rev. 1998;11(3):480-96.

5. Almas A, Parkash O, Akhter J. Clinical factors associated with mortality in dengue infection at a tertiary care center. Southeast Asian J Trop Med Public Health. 2010;41(2):333-40.

6. Hasan S, Jamdar SF, Alalowi M, Al Ageel Al Beaiji SM. Dengue virus: a global human threat: review of literature. J Int Soc Prev Community Dent. 2016; 6(1):1-6.

7. Weeratunga PN, Caldera MC, Gooneratne IK, Gamage R, Perera P. Neurological manifestations of dengue: a cross sectional study. Travel Med Infect Dis. 2014;12(2):189-93.

8. Shivanthan MC, Ratnayake EC, Wijesiriwardena BC, Somaratna KC, Gamagedara LK. Paralytic squint due to abducens nerve palsy: a rare consequence of dengue fever. BMC Infect Dis. 2012;12:156.

9. Mazliha M, Boo YL, Chin PW. Isolated unilateral sixth cranial nerve palsy: a rare presentation of dengue fever. Malays Fam Physician. 2016;11(1):25-6.

10. Mangold KA, Reynolds SL. A review of dengue fever: a resurging tropical disease. Pediatr Emerg Care. 2013;29(5):665-9. quiz 670-1

11. Carod-Artal FJ, Wichmann O, Farrar J, Gascon J. Neurological complications of dengue virus infection. Lancet Neurol. 2013;12(9):906-19.

12. Ministry of Health, Sri Lanka. Guidelines on management of dengue fever \& dengue haemorrhagic fever in adults. Colombo: Ministry of Health, Sri Lanka; 2012.

13. Verma R, Sharma P, Garg RK, Atam V, Singh MK, Mehrotra HS. Neurological complications of dengue fever: experience from a tertiary center of north India. Ann Indian Acad Neurol. 2011;14(4):272-8.

14. Goswami RP, Mukherjee A, Biswas T, Karmakar PS, Ghosh A. Two cases of dengue meningitis: a rare first presentation. J Infect Dev Ctries. 2012;6(2): 208-11.

15. Misra UK, Kalita J, Syam UK, Dhole TN. Neurological manifestations of dengue virus infection. J Neurol Sci. 2006;244(1-2):117-22.

16. Jackson ST, Mullings A, Bennett F, Khan C, Gordon-Strachan G, Rhoden T. Dengue infection in patients presenting with neurological manifestations in a dengue endemic population. West Indian Med J. 2008;57(4):373-6.

17. Ralapanawa DM, Kularatne SA, Jayalath WA. Guillain-Barré syndrome following dengue fever and literature review. BMC Res Notes. 2015;8:729.

18. Boo YL, Aris MAM, Chin PW, Sulaiman WAW, Basri H, Hoo FK. Guillain-Barré syndrome complicating dengue fever: two case reports. Ci Ji Yi Xue Za Zhi. 2016;28(4):157-9.

19. Sanjay S, Wagle AM, Au Eong KG. Optic neuropathy associated with dengue fever. Eye (Lond). 2008;22(5):722-4.

20. Sanguansermsri T, Poneprasert B, Phornphutkul B, Kulapongs P, Tantachamrun T. Acute encephalopathy associated with dengue infection. Bangkok, Thailand: SEAMEO TROPMED; 1976. p. 10-1.

21. Murthy JM. Neurological complication of dengue infection. Neurol India. 2010;58(4):581-4. 
22. Berlit P. Diagnosis and treatment of cerebral vasculitis. Ther Adv Neurol Disord. 2010;3(1):29-42.

23. Solomon T, Dung NM, Vaughn DW, Kneen R, Thao LT, Raengsakulrach B, Loan HT, Day NP, Farrar J, Myint KS, et al. Neurological manifestations of dengue infection. Lancet. 2000;355(9209):1053-9.

24. Nanda SK, Jayalakshmi S, Mohandas S. Pediatric ischemic stroke due to dengue vasculitis. Pediatr Neurol. 2014;51(4):570-2.

25. Li GH, Ning ZJ, Liu YM, Li XH. Neurological manifestations of dengue infection. Front Cell Infect Microbiol. 2017;7:449.

26. Verma R, Sharma P, Khurana N, Sharma LN. Neuralgic amyotrophy associated with dengue fever: case series of three patients. J Postgrad Med. 2011;57(4): 329-31.

27. Gupta M, Nayak R, Khwaja GA, Chowdhury D. Acute disseminated encephalomyelitis associated with dengue infection: a case report with literature review. J Neurol Sci. 2013;335(1-2):216-8.

28. Fong CY, Hlaing CS, Tay CG, Kadir KA, Goh K, Ong LC. Longitudinal extensive transverse myelitis with cervical epidural haematoma following dengue virus infection. Eur J Paediatr Neurol. 2016;20(3):449-53.

29. Mishra A, Shukla S, Aggarwal S, Chaudhary B. Lateral rectus palsy in a case of dengue fever. Med J Armed Forces India. 2015;71(Suppl 1):S101-3.

30. Donnio A, Beral L, Olindo S, Cabie A, Merle H. Dengue, a new etiology in oculomotor paralysis. Can J Ophthalmol. 2010;45(2):183-4.

31. Peter S, Malhotra N, Peter P, Sood R. Isolated Bell's palsy - an unusual presentation of dengue infection. Asian Pac J Trop Med. 2013;6(1):82-4.

\section{Submit your next manuscript to BioMed Central and we will help you at every step:}

- We accept pre-submission inquiries

- Our selector tool helps you to find the most relevant journal

- We provide round the clock customer support

- Convenient online submission

- Thorough peer review

- Inclusion in PubMed and all major indexing services

- Maximum visibility for your research

Submit your manuscript at www.biomedcentral.com/submit 